\title{
El uso de aspirina a largo plazo prevendría la incidencia de cáncer colorrectal
}

Effect of aspirin on long-term risk of colorectal cancer

\section{Objetivo}

Revisar el efecto del uso de aspirina para reducir la incidencia de cáncer colorrectal (CR).

\section{Diseño}

Revisión no sistemática de dos ensayos clínicos controlados aleatorizados (ECCA) y revisión sistemática de estudios observacionales (cohortes $n=11$ y casos-control $n=19$ ) con un seguimiento de hasta 20 años.

\section{Selección de estudios}

Los estudios observacionales fueron seleccionados de forma sistemática a través de PubMed, no reportándose el modo de selección de los ECCA.

\section{Medición de resultados principales \\ Incidencia de CR.}

\section{Resultados principales}

Ambos ECCA mostraron una reducción en la incidencia de CR con el uso de aspirina durante cinco o más años (ver tabla 1) luego de luego de diez años de iniciado el tratamiento (años 0 a 9: 0,92 IC95\% 0,56 a 1,49; años 10 a 19: 0,60 IC95\% 0,42 a 0,87 ) fue dependiente de la duración y de la adherencia al tratamiento. Fue más pronunciado entre los años 10 a 14 en quienes la usaron al menos durante cinco años (0,37 IC95\% $0,20$ a 0,70$)$ y en quienes tuvieron buena adherencia $(0,26$ IC95\% 0,12 a 0,56).
Flossmann E et al. Lancet 2007 May 12; 369:1603-13

En los estudios de casos $(n=20.815)$ y controles y de cohortes, luego de ajustar por edad, sexo, raza, historia familiar, localización y agresividad del cáncer; el uso regular de $300 \mathrm{mg} o$ más de aspirina (u otros AINE) durante más de diez años se asoció con una reducción del riesgo de $\mathrm{CR}$.

Tabla 1: efecto del uso de aspirina en la incidencia de cáncer colorrectal (estudios aleatorizados con más de 20 años de seguimiento).

\begin{tabular}{l|c|c|c|c}
\hline \multirow{2}{*}{ British Doctors Aspirin Trial' $^{1}$} & \multicolumn{2}{|c|}{$\begin{array}{c}\text { Casos de cancer } \\
\text { colorectal }\end{array}$} & \multirow{2}{*}{$\begin{array}{c}\text { Hazard ratio } \\
\text { (IC95\%) }\end{array}$} & NNT \\
\cline { 2 - 3 } & Aspirina & Gontrol & & \\
\hline UK-TIA trial $^{2}$ & $92 / 3.429$ & $64 / 1.710$ & $0,70(0,51-0,97)$ & 100 \\
\hline Resultados Combinados & $37 / 1.632$ & $23 / 817$ & $0,82(0,49-1,38)$ & 117 \\
\hline
\end{tabular}

\section{Conclusiones}

300mg de aspirina por día durante un mínimo de cinco años serían efectivos para prevenir el CR con una latencia aproximada de diez años.

Palabras clave: revisión sistemática, aspirina, cáncer de colon, prevención. Key words: systematic review, aspirin, colonic cancer, prevention.

Fuente de Financiamiento/conflicto de interés de los autores: Rothwell recibió financiamiento de varias compañías farmacéuticas para conferencias y comités de estudios clínicos (Sanofi-BMS, Servier, Bayer y AstraZeneca) pero se declara sin conflictos de interés.

\section{Comentario}

Este estudio muestra que la aspirina en dosis más altas que las que se usan para prevenir enfermedades cardiovasculares, reduciría el riesgo de $C R$.

Presenta sin embargo algunas limitaciones: 1) basa sus conclusiones en una revisión no sistemática (sesgo de selección*) de dos estudios aleatorizados hechos en el Reino Unido; 2) uno de ellos no usó tratamiento en su grupo control'; 3) ninguno fue diseñado para detectar incidencia* de CR en sus resultados principales $^{1,2} ; 4$ ) tampoco se contemplaron los efectos adversos relacionados al uso de aspirina.

Por otra parte, este trabajo es concordante con otra revisión sistemática hecha por la Agencia de Investigación y Calidad de los Servicios de Salud ${ }^{3}$ de EEUU (en inglés HRQA). Sus conclusiones demuestran resultados similares, pero con la adición de una revisión sistemática de los efectos adversos del uso de aspirina. La Fuerza de Tareas Norteamericana se expide en contra del uso rutinario de aspirina y AINE para la prevención del CR en la población general ${ }^{4}$ ya que a pesar de la evidencia a favor de su uso, el riesgo de hemorragia gastrointestina (dosis-dependiente) y ACV hemorrágico son altos.

\section{Conclusiones del comentador}

Si bien el uso en la población general de altas dosis de aspirina durante más de diez años reduciría el riesgo de adquirir cáncer $\mathrm{CR}$, sus potenciales efectos adversos la hacen una práctica riesgosa y no recomendada rutinariamente.

Gabriela Lewin [ Médica de familia Kemptville District Hospital, Universidad de Ottawa, Canadá. ]

Lewin G. El uso de aspirina a largo plazo prevendría la incidencia de cáncer colorrectal. Evid. actual. práct. ambul; 10(4): 102, Jul-Ago.2007. Effect of aspirin on long-term risk of colorectal cancer: consistent evidence from randomized and observational studies. Flossmann $E$ et al. Lancet. 2007 May 12; 369:1603-13. PMID: 17499602. Disponible en URL con suscripción http://www.ncbi.nIm.nih.gov/sites/entrez?Db=pubmed\&Cmd=ShowDetailView\&TermToSearch=17499581

\section{Referencia}

1. Randomized trial of prophylactic daily aspirin in British male doctors. Peto R, Gray R, Collins R y col. BMJ 1988; 296:313-16

2. The United Kingdom transient ischemic attack (UK-TIA) aspirin trialN final results. Farrel B, Godwin J, Richards S y col. J Neurol Neurosurg Psychiatry. 1991; 54 : $1044-54$ 3. The use of aspirin for primary prevention of colorectal cancer: a systematic review prepared for the US Preventive Services Task Force. Dubé C, Rostom A, Lewin G y col. Ann Intern Med. 2007; 146:365-375.

4. Rutine aspirin or nonsteroidal anti-inflammatory drugs for the primary prevention of colorectal cancer: US Preventive Services Task Force Recommendation Statement. Ann Intern Med. 2007:146:361-364. 